\section{Case Reports in Ophthalmology}

Case Rep Ophthalmol 2020;11:473-480

DOI: 10.1159/000509337

Published online: August 11, 2020
(C) 2020 The Author(s)

Published by S. Karger AG, Basel www.karger.com/cop

This article is licensed under the Creative Commons Attribution-NonCommercial 4.0 International License (CC BY-NC) (http://www.karger.com/Services/OpenAccessLicense). Usage and distribution for commercial purposes requires written permission.

\title{
Suspected Gentamicin-Induced Retinal Vascular Occlusion after Vitrectomy
}

\author{
Fumiko Murao ${ }^{a}$ Takamasa Kinoshita $^{\mathrm{b}}$ Takashi Katome $^{\mathrm{a}}$ Hiroki Sano $^{\mathrm{a}}$ \\ Masanori Niki ${ }^{a}$ Yoshinori Mitamura ${ }^{a}$ \\ aDepartment of Ophthalmology, Institute of Biomedical Sciences, Tokushima University \\ Graduate School, Tokushima, Japan; 'bepartment of Ophthalmology, Sapporo City \\ General Hospital, Sapporo, Japan
}

\section{Keywords}

Gentamicin · Macular ischemia · Retinal vascular occlusion · Vitrectomy

\begin{abstract}
Retinal vascular occlusion after ocular surgery is a rare but serious complication. A history of cardiovascular diseases, retrobulbar anesthesia injection, high intraocular pressure during the perioperative period, and drug toxicity have been reported as possible causative factors. We report here two cases of multiple retinal vascular occlusions after the subconjunctival injection of gentamicin at the end of uncomplicated 25-gauge vitrectomy. Case 1 was a 61-year-old man who developed a macular hole in the right eye. Phacovitrectomy with gas tamponade was performed. On postoperative day (POD) 1, dot hemorrhage was observed on the temporal side of the optic disk. On POD10, macular whitening, retinal hemorrhage, and multiple occlusion of retinal arteries and veins were observed. Case 2 was a 51-year-old woman who was diagnosed with rhegmatogenous retinal detachment in the right eye and underwent phacovitrectomy with gas tamponade. On POD3, macular whitening with cotton wool spots and retinal hemorrhage were observed with macular ischemia owing to occlusion of retinal arteries and veins. In both cases, subconjunctival injection of gentamicin given at the end of surgery was the most suspected cause of retinal vascular occlusion.




\section{Case Reports in Ophthalmology}

\section{Introduction}

A history of cardiovascular disease, retrobulbar anesthesia injection, high intraocular pressure (IOP) during the perioperative period, and drug toxicity have been reported as the possible causative factors of the rare and serious complication of retinal vascular occlusion after intraocular surgery [1-3].

We herein report two cases of multiple retinal vascular occlusion after the subconjunctival injection of gentamicin at the end of 25 -gauge $(25 \mathrm{G})$ vitrectomy.

\section{Case Presentation}

Case 1

A 61-year-old man visited our department with a complaint of decreased visual acuity of the right eye for 1 month. A stage 2 macular hole in the right eye was noted (Fig. 1a, b). His decimal best-corrected visual acuity (BCVA) at the first visit was 0.3 in the right eye and 1.0 in the left eye. Furthermore, his IOP was $15 \mathrm{~mm} \mathrm{Hg}$ in the right eye and $14 \mathrm{~mm} \mathrm{Hg}$ in the left eye. The axial length was $23.28 \mathrm{~mm}$ in the right eye and $23.44 \mathrm{~mm}$ in the left eye. No systemic diseases such as diabetes or hypertension were observed. Accordingly, phacovitrectomy with a 20\% sulfur hexafluoride (SF6) gas tamponade was performed under retrobulbar anesthesia. At the end of the operation, a subconjunctival injection of gentamicin was administered. Dot hemorrhages were observed on the temporal side of the optic disk on postoperative day (POD) 1. However, neither further hemorrhage nor retinal whitening was observed on POD3, and the patient was discharged. Fundus examination on POD10 revealed blot hemorrhage, mottled retinal whitening in the macula, and cotton wool spots. The patient's BCVA was 0.01 in the right eye and decreased to hand motion during postoperative week 1 . Fundus examination and fluorescein angiography (FA) showed multiple disruptions of the vessels surrounding the fovea and occlusion of the macular branch retinal artery on the inferior temporal side. Slight fluorescence leakage on the nasal side of the optic nerve head was observed (Fig. 1c, e). Limaprost alfadex and kallidinogenase were prescribed as antiplatelet and vasodilator therapy, along with an oral steroid. Stellate ganglion block was performed to increase ocular circulation. Progression of arteriovenous occlusion around the macula was observed. The macular hole was finally closed, but the patient's postoperative BCVA was 0.2 .

\section{Case 2}

A 51-year-old woman presented with complaints of blurred vision lasting for 6 days. Her decimal BCVA at the first visit was 0.02 in the right eye and 1.2 in the left eye. Her IOP was 13 $\mathrm{mm} \mathrm{Hg}$ in the right eye and $15 \mathrm{~mm} \mathrm{Hg}$ in the left eye. Mild cataract was observed in both eyes. The axial length was $26.07 \mathrm{~mm}$ in the right eye and $25.88 \mathrm{~mm}$ in the left eye. Bullous maculaoff retinal detachment was observed in the right eye, with retinal tears in the superior area and temporal inferior area of the retina (Fig. 2a). No systemic diseases such as diabetes or hypertension were observed. Under retrobulbar anesthesia, phacovitrectomy with a $20 \%$ SF 6 gas tamponade was performed. At the end of the operation, a subconjunctival injection of gentamicin was administered. On POD1, subretinal fluid persisted in the macula, but it was almost absorbed on POD2. Dot bleedings and retinal whitening were observed in the macula on POD3. Blot and flame-shaped hemorrhage and cotton wool spots were observed on POD4 (Fig.

$2 \mathrm{~b}, \mathrm{~d}$ ). FA showed multiple retinal vein filling defects around the macula and tissue staining of the vascular wall. A small nonperfused region was also observed near the laser coagulation 


\section{Case Reports in Ophthalmology}

Case Rep Ophthalmol 2020;11:473-480 DOI: $10.1159 / 000509337$

c) 2020 The Author(s). Published by S. Karger AG, Basel www.karger.com/cop

spots (Fig. 2c). The patient's BCVA was $50 \mathrm{~cm}$ hand motion. The patient was treated with urokinase infusion and oral acetazolamide, but there was no significant improvement in retinal circulation. The retina was reattached. Two months after the operation, her visual acuity was 0.08 . There was a central vision defect 16 months after the operation, but local reperfusion of the retinal circulation occurred and the visual acuity improved to 0.8 . Within 1.5 months postoperatively, some of the blood vessels located proximal to the optic nerve were reperfused. The distally located blood vessels showed progressive occlusion (Fig. 3a-c). A reperfusion of blood vessels was observed on the nasal superior side of the macula. Optical coherence tomography angiography 1 year after surgery showed a highly damaged capillary network on the superficial capillary plexus (Fig. 3d) and deep capillary plexus (Fig. 3e). The capillaries superior to the macula were less damaged than those inferior to the macula. In visual field examination, a small visual field remained on the temporal inferior side of the central scotoma (Fig. 3f).

Regarding $25 \mathrm{G}$ phacovitrectomy in these two cases, we used a disposable retrobulbar needle for retrobulbar anesthesia. The anesthetic used was a 1:1 mixture of 4-5 mL mepivicaine ( $2 \%$ Carbocaine $\left.^{\circledR}\right)$ and bupivacaine $\left(0.5 \%\right.$ Marcaine $\left.^{\circledR}\right)$. Compression of the ocular globe was not performed after injection. After phacoemulsification with intraocular lens implantation, the patients underwent 3-port (case 1) and 4-port (case 2) vitrectomy. We used singleuse phaco tips, I/A tips, $25 \mathrm{G}$ cutters, and probes. No ethylene oxide gas-sterilized instruments were used. The IOP control system was set from 25 to $30 \mathrm{~mm} \mathrm{Hg}$ and was reduced to $15 \mathrm{~mm}$ $\mathrm{Hg}$ during ocular globe compression. Triamcinolone acetonide was used for vitreous staining. Internal limiting membrane peeling was performed in case 1, and retinal photocoagulation around the retinal breaks was performed in case 2. The SF6 gas amount was manually adjusted to $20 \%$ before injection. Next, the wound was sutured, and the pressure was confirmed to be at physiological level by digital palpation. At the end of the surgery, subconjunctival injection of gentamicin sulfate $(5 \mathrm{mg}$ in $0.5 \mathrm{~mL})$ and dexamethasone $(0.8 \mathrm{mg}$ in $0.5 \mathrm{~mL})$ was performed. The total operation time was $40 \mathrm{~min}$ for case 1 and $80 \mathrm{~min}$ for case 2 . There were no intraocular fluctuations in IOP. Postoperatively, both patients were instructed to maintain the prone position.

In both cases, no vascular abnormalities in the head and neck were found on postoperative magnetic resonance imaging and magnetic resonance angiography, and there were no underlying systemic cardiovascular disorders.

\section{Discussion/Conclusion}

We report two cases of multiple retinal vascular occlusions after the subconjunctival injection of gentamicin at the end of $25 \mathrm{G}$ vitrectomy.

In recent years, there have been reports of subconjunctival gentamicin-induced macular toxicity following sutureless $25 \mathrm{G}$ vitrectomy [4-6]. Fundus examination showed retinal hemorrhages, edema, and soft exudates on the posterior pole on POD1 [4] or POD3 [5]. In another report, retinal pallor throughout the macula with a macular hole was observed on POD14 [6]. Conway et al. [7] reported on gentamicin-induced retinal toxicity in the primate retina by injecting gentamicin into the vitreous cavity of Cebus navrigatus monkeys. According to the report, gentamicin causes inflammatory changes in the inner layer of the retina in the posterior pole, especially in the nerve fiber layer and the ganglion cell layer. Vascular occlusion occurs secondly owing to granulocyte plug formation around the capillary bed. In the present cases, optical coherence tomography images obtained when retinal opacification appeared showed 


\section{Case Reports in Ophthalmology}

Case Rep Ophthalmol 2020;11:473-480 DOI: $10.1159 / 000509337$

(c) 2020 The Author(s). Published by S. Karger AG, Basel www.karger.com/cop

Murao et al.: Multiple Retinal Vascular Occlusion after Vitrectomy

hyperreflectivity in the inner retinal layers (Fig. 1d and 2e). FA in both cases showed nonfilling of the retinal capillaries in the central macula (Fig. 1e and 2c). The distinctive angiographic appearance of shutdown of the capillary bed observed may be the result of irreversible "granulocyte plugging" after the vessel is reopened [7].

Wound leak and hypotony promotes exchange of conjunctival fluid with the vitreous fluid, and shaving of the peripheral vitreous tends to cause hypotony owing to insufficient wound closure. In our cases, the wound was sutured, and the pressure was confirmed to be at physiological level by digital palpation before injection of subconjunctival gentamicin. The IOP was within normal tension on the day after operation. However, it is impossible to rule out the possibility that a small amount of gentamicin had flowed into the eye because of insufficiently sutured wounds. Furthermore, a small amount of drug that would not normally cause toxicity may cause retinal damage, as the SF6 gas-filled vitreous cavity contains almost no liquid component [8].

In case 2, although the central scotoma remained 16 months after the operation, visual acuity improved to 0.8 . The reperfusion of capillaries in the nasal superior side of the macula may have improved visual field and visual acuity (Fig. 3) [8].

Other than drug toxicity, postoperative vascular occlusion is caused by post-bulbar anesthesia, gas injection, or increased IOP during and after surgery [1-3]. In our case, no obvious IOP abnormality was observed during or after surgery, and no problems such as retrobulbar hemorrhage occurred during retrobulbar anesthesia. Fundus findings are different from those of typical central retinal artery occlusion and central retinal vein occlusion [9].

Retinal vascular occlusion after vitrectomy is rare but can be a serious complication that can lead to severe visual impairment. The risks of prophylactic use of subconjunctival injection of gentamicin should be considered, given that no evidence has been reported that prophylactic subconjunctival injection of antibacterial agents can prevent endophthalmitis after vitrectomy [10]. Gentamicin injected under the conjunctiva may flow into the vitreous cavity and induce retinal vascular occlusion, even if all ports are sutured.

\section{Acknowledgements}

The authors thank E. Morishita for assistance with ophthalmic examination.

\section{Statement of Ethics}

This case report was conducted ethically in accordance with the World Medical Association Declaration of Helsinki. Written informed consent was obtained from the patients. This study does not include any information which may reveal the patient's identity.

\section{Disclosure Statement}

The authors have no conflicts of interest to declare. 


\section{Case Reports in Ophthalmology}

Funding Sources

No funding was received.

\section{Author Contributions}

All authors participated in the analysis of both cases. All authors reviewed and approved the manuscript.

\section{References}

1 Fischer C, Bruggemann A, Hager A, Callizo Planas J, Roider J, Hoerauf H. Vascular occlusions following ocular surgical procedures: a clinical observation of vascular complications after ocular surgery. J Ophthalmol. 2017;2017:9120892.

2 Tappeiner C, Garweg JG. Retinal vascular occlusion after vitrectomy with retrobulbar anesthesiaobservational case series and survey of literature. Graefes Arch Clin Exp Ophthalmol. 2011 Dec;249(12):1831-5.

3 Russell JF, Scott NL, Haddock LJ, Eaton AM, Flynn HW. Central retinal artery occlusion on postoperative day one after vitreoretinal surgery. Am J Ophthalmol Case Rep. 2018 Oct;12:93-6.

4 Cardascia N, Boscia F, Furino C, Sborgia L. Gentamicin-induced macular infarction in transconjunctival sutureless 25-gauge vitrectomy. Int Ophthalmol. 2008 Oct;28(5):383-5.

5 Brouzas D, Moschos MM, Koutsandrea C, Davou S, Georgalas I. Gentamicin-induced macular toxicity in 25gauge sutureless vitrectomy. Cutan Ocul Toxicol. 2013 Sep;32(3):258-9.

6 Heath Jeffery RC, Bowden FJ, Essex RW. Subconjunctival gentamicin-induced macular toxicity following sutureless 25-gauge vitrectomy. Clin Exp Ophthalmol. 2017 Apr;45(3):301-4.

7 Conway BP, Tabatabay CA, Campochiaro PA, D'Amico DJ, Hanninen LA, Kenyon KR. Gentamicin toxicity in the primate retina. Arch Ophthalmol. 1989 Jan;107(1):107-12.

8 Campochiaro PA, Lim JI; The Aminoglycoside Toxicity Study Group. Aminoglycoside toxicity in the treatment of endophthalmitis. Arch Ophthalmol. 1994 Jan;112(1):48-53.

9 Hayreh SS. Central retinal artery occlusion. Indian J Ophthalmol. 2018 Dec;66(12):1684-94.

10 Weiss SJ, Adam MK, Gao X, Obeid A, Sivalingam A, Fineman MS, et al. Endophthalmitis after pars plana vitrectomy: efficacy of intraoperative subconjunctival antibiotics. Retina. 2018 Sep;38(9):1848-55. 


\section{Case Reports in Ophthalmology}
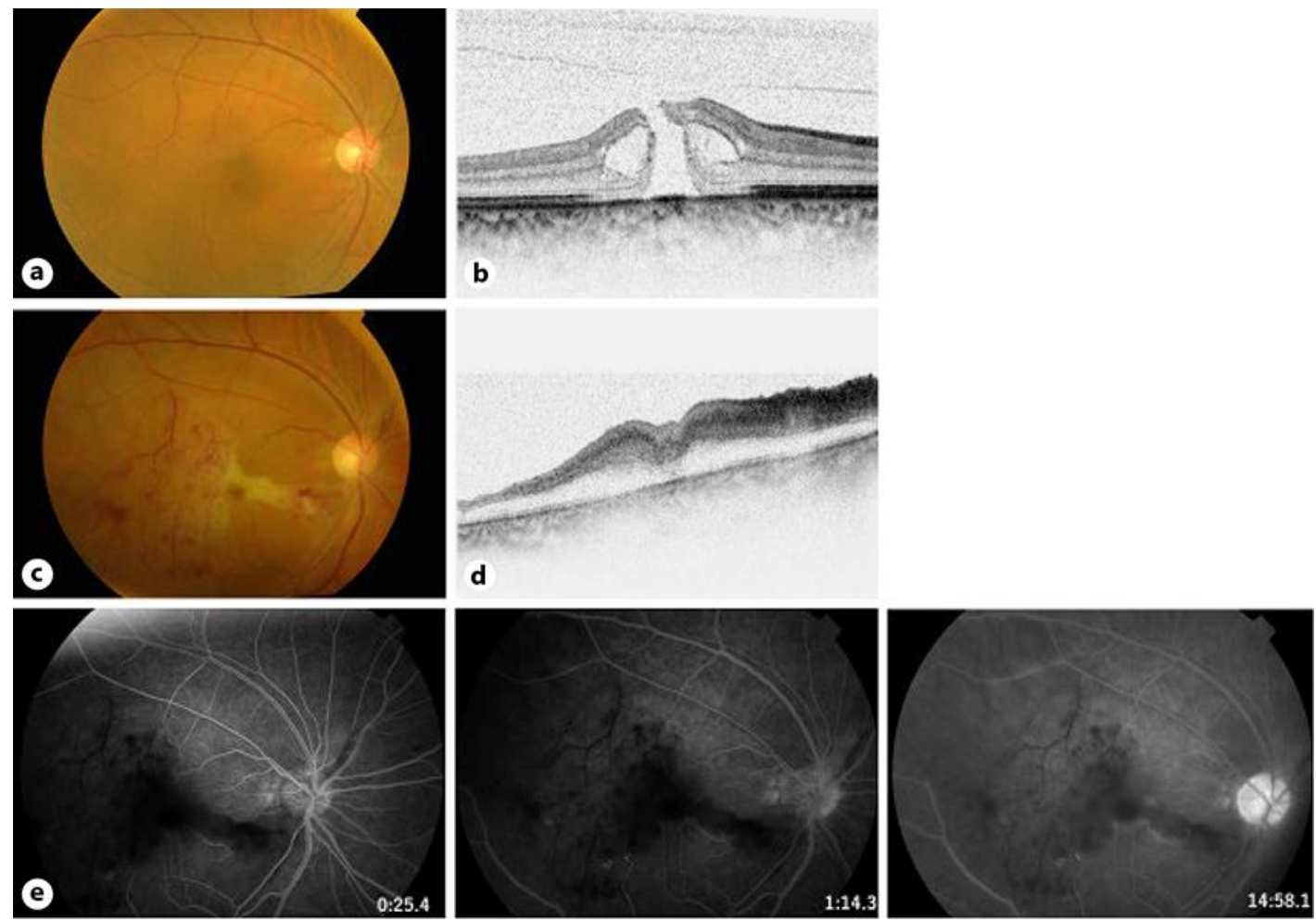

Fig. 1. Multimodal imaging including color fundus photography, optical coherence tomography, and FA of case 1 showing a macular hole in the right eye. a Preoperative fundus photograph showing a macular hole. There is no apparent vascular arteriosclerosis. b Preoperative horizontal optical coherence tomography showing a stage 2 macular hole with cysts at the edge of the hole. c Fundus photography 17 days after vitrectomy showing mottled whitening of the macular retina, cotton wool spots, and blot hemorrhages. There are multiple disruptions of the vessels surrounding the fovea. $\mathbf{d}$ Horizontal optical coherence tomography through the fovea 17 days after vitrectomy demonstrating thickening and hyperreflectivity of the inner retinal layers. e FA 25 days after vitrectomy showing a nonperfusion area with filling defect in the macular branch of the inferotemporal branch retinal artery and multiple macular arterial occlusion around the fovea. No retinal capillary filling in the central part of the macular even in the late phase was observed. 


\section{Case Reports in Ophthalmology}
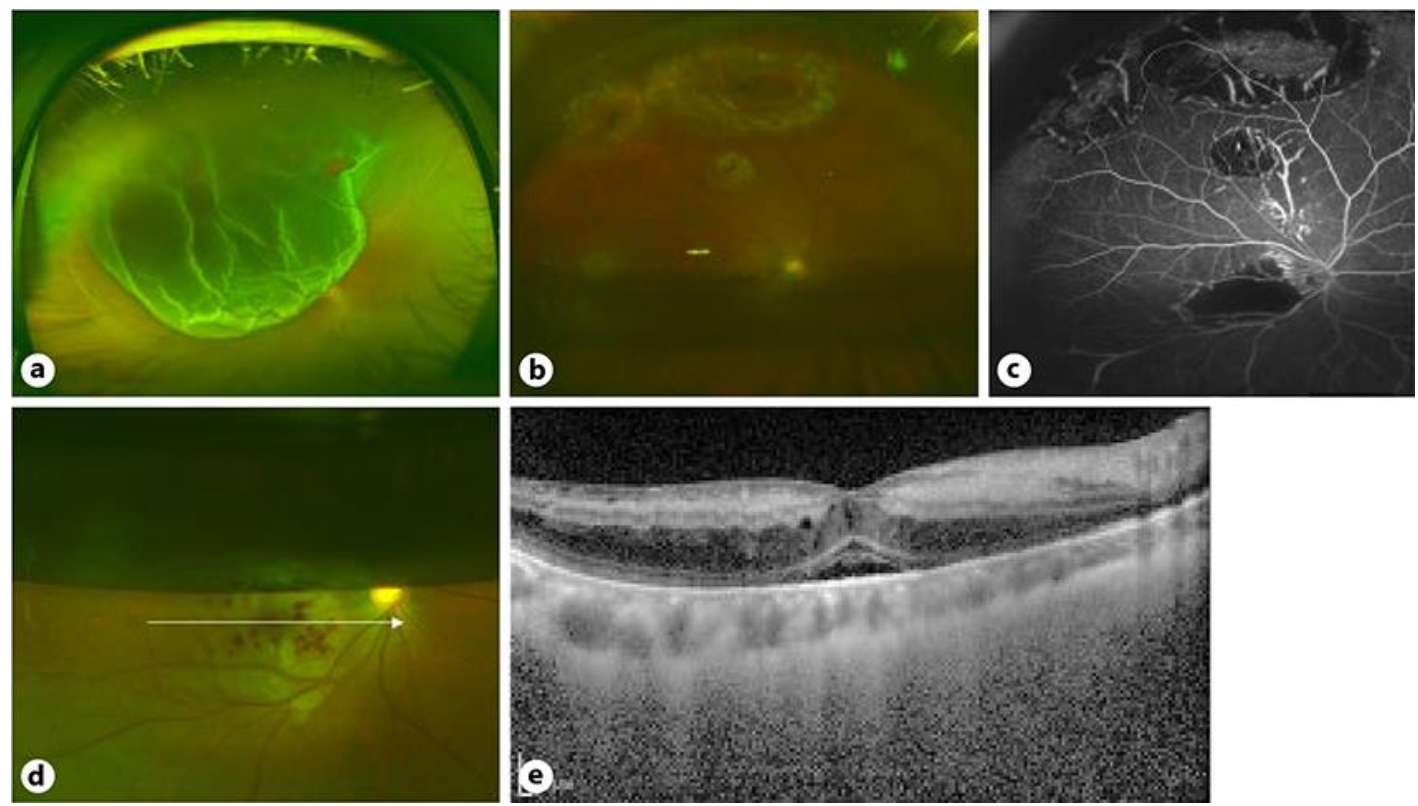

Fig. 2. Multimodal imaging including color fundus photography, optical coherence tomography, and FA of case 2 showing rhegmatogenous retinal detachment in the right eye. a Preoperative fundus photograph showing the bullous macula-off retinal detachment. b Fundus photograph 4 days after vitrectomy showing macular whitening and retinal hemorrhage. Half of the $20 \%$ sulfur hexafluoride gas remains. There are two breaks on the upper side, an intentional break below them, and laser photocoagulation spots around these breaks. c FA 4 days after vitrectomy showing an avascular area around the macula and inferior to the intentional break. $\mathbf{d}$ Fundus photography 6 days after vitrectomy showing blot retinal hemorrhage, retinal whitening, and cotton wool spots. e Optical coherence tomography through the white arrow in d demonstrating thickening and hyperreflectivity of the inner retinal layers and subfoveal retinal detachment. 


\section{Case Reports in Ophthalmology}
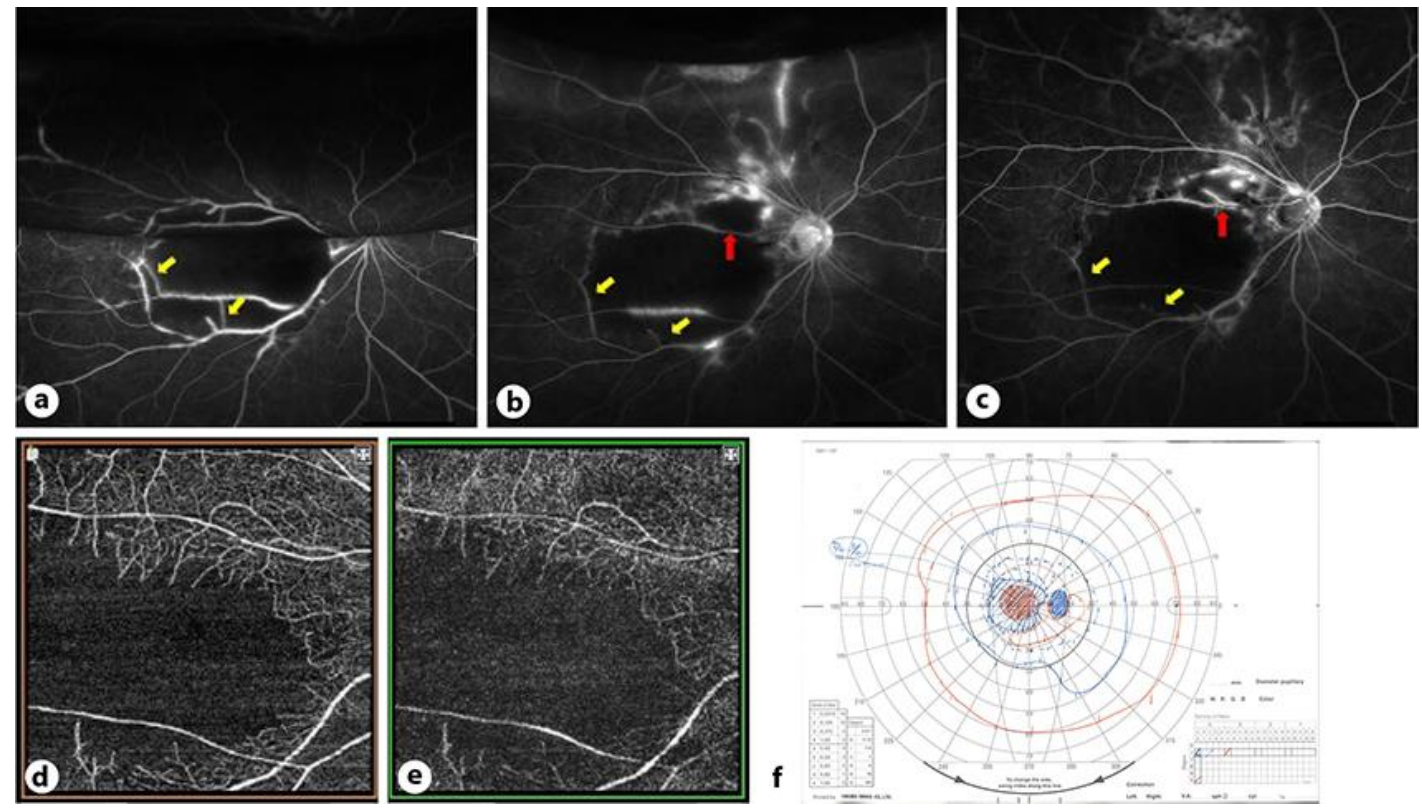

Fig. 3. FA and optical coherence tomography angiography of case 2. a FA on postoperative day 6 showing multiple retinal vascular filling defects around the macula. There is staining of the arcade vessel wall. The superior half is a mirror image owing to intraocular gas. b FA on postoperative day 12. Supratemporal vessels to the optic disc also show filling defect. c FA on postoperative day 45. The occluded blood vessels located proximal to the optic nerve are reperfused (red arrow), whereas the occlusion of distally located blood vessels have progressed (yellow arrows). d, e Optical coherence tomography angiography 1 year after surgery showing a highly damaged capillary network on the superficial capillary plexus (d) and deep capillary plexus (e). The capillaries superior to the macula are less damaged than those inferior to the macula. $f$ Visual field of the right eye 16 months after the operation. Partial visual field remains on the lower ear side of the central scotoma. 